\title{
Different Roles of TP53 Codon 72 Polymorphism in Type 2 Diabetes and Its Complications: Evidence from a Case-Control Study on a Chinese Han Population
}

\author{
Dong Guo' \\ Liang Fang ${ }^{2}$ \\ Xuelai $\mathrm{Yu}^{3}$ \\ Congyang Wang ${ }^{4}$ \\ Yan Wang ${ }^{4}$ \\ Wenwen Guo iD ${ }^{4}$ \\ 'Department of Gastrointestinal Surgery, \\ The Affiliated Hospital of Qingdao \\ University, Qingdao, 266000, People's \\ Republic of China; ${ }^{2}$ Department of \\ Gastroenterology, The Affiliated Hospital \\ of Qingdao University, Qingdao, 266000, \\ People's Republic of China; ${ }^{3}$ Department \\ of Neurology, The Second Affiliated \\ Hospital of Nanjing Medical University, \\ Nanjing, 2100II, People's Republic of \\ China; ${ }^{4}$ Department of Pathology, The \\ Second Affiliated Hospital of Nanjing \\ Medical University, Nanjing, 2100II, \\ People's Republic of China
}

Objective: The purpose of this study was to investigate the relationships between TP53 Pro72Arg (rs1042522) polymorphism and susceptibility to type 2 diabetes (T2DM) and its related complications.

Methods: The TP53 Pro72Arg polymorphism was genotyped by polymerase chain reactionrestriction fragment length polymorphism (PCR-RFLP) method in 206 T2DM patients and 446 healthy controls. Mitochondrial DNA (mtDNA) content, mtDNA transcriptional level and large-scale mtDNA deletion were evaluated in leukocytes of T2DM patients using fluorescence-based quantitative PCR (FQ-PCR), reverse transcriptase-quantitative PCR (RTqPCR) and long-range PCR approaches, respectively. The data of our study were processed by GraphPad Prism software (version 7.00).

Results: The distribution of TP53 Pro72Arg differed in T2DM patients from the controls, with a moderately increased proportion of TP53 Arg72 variant carriers (Pro/Arg and Arg/Arg genotypes) $(88.3 \%$ vs $81.2 \%, p=0.022 ; \mathrm{OR}=1.089,95 \% \mathrm{CI}=1.018-1.164)$. T2DM patients with $\mathrm{Arg} /$ Arg genotype had significantly decreased prevalences of diabetic neuropathy and retinopathy compared to those without $(6.5 \%$ vs $19.4 \%, p=0.018$ and $14.8 \%$ vs $30.7 \%, p=0.018$, respectively). T2DM patients with $\mathrm{Arg} / \mathrm{Arg}$ genotype had higher mtDNA content and mtRNA expression level than those who were not $\operatorname{Arg} / \operatorname{Arg}$ genotype ( $p<0.05$ for all), and we did not observe mtDNA 4977-base pair (bp) deletion mutations in the leukocytes of T2DM patients.

Conclusion: There was a significant association of the TP53 Pro72Arg polymorphism with susceptibility to T2DM, and the homozygous Arg/Arg genotype of this gene locus might be a protective factor for diabetic complications. Those results suggested that the TP53 Arg72 variant had a different association with type 2 diabetes and its complications, and it might be related to mtDNA maintenance of the TP53 Arg72 variant under hyperglycemia-induced stress.

Keywords: TP53, type 2 diabetes mellitus, complication, mtDNA content, mtRNA, mtDNA maintenance

\section{Introduction}

Diabetes mellitus (DM) is a lifelong disease, and it remains an extremely important public health problem because of its high prevalence and serious complications in the whole world. ${ }^{1}$ There are two principal forms of diabetes, type 1 and type 2 diabetes mellitus, ${ }^{1}$ of which type 2 diabetes (T2DM), non-insulin-dependent diabetes, is a set of dysfunctions characterized by hyperglycemia resulting from insulin resistance and/or insufficient insulin secretion from pancreatic $\beta$-cells, ${ }^{2-4}$ and it accounts for roughly $90 \%$ of all cases worldwide. ${ }^{1,3}$ 


\section{Graphical Abstract}
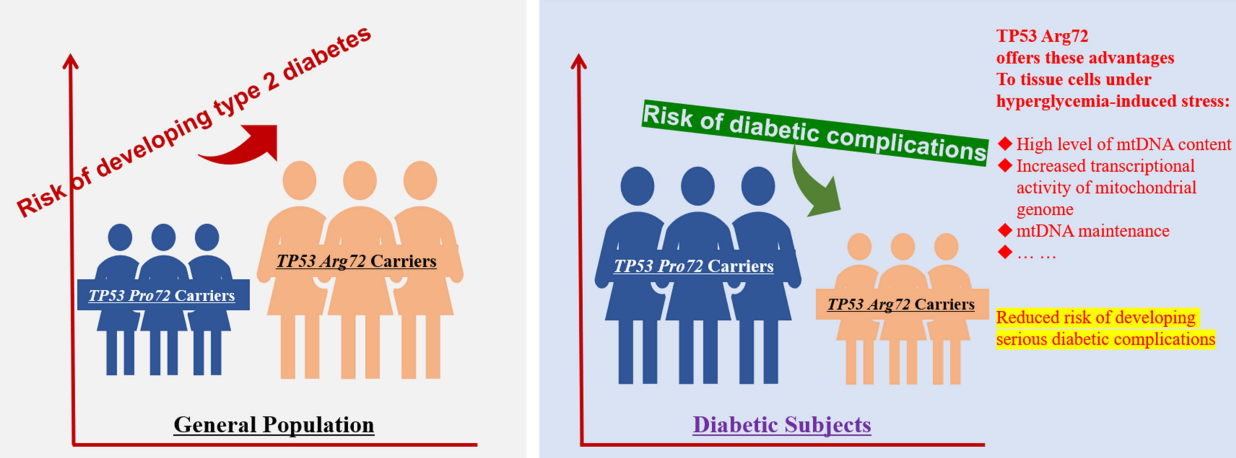

Evidence has revealed that tumor suppressor protein p53 (TP53) plays an important role in developing DM, because it is involved in regulating insulin resistance of target tissues $^{5}$ and $\beta$-cell dysfunction. ${ }^{6}$ In human, a common polymorphism of $C C C / C G C$ base transversion at the codon 72 of TP53 gene causes a change of a proline residue (CCC, Pro72) to an arginine residue (CGC, Arg72), and is named TP53 Pro72Arg (p.Pro72Arg) polymorphism. The TP53 Pro72Arg polymorphism is located in the functional region of proline-rich domain that can cause alterations of TP53 biological function. ${ }^{7}$ Functional analyses showed that the TP53 $\operatorname{Arg} 72$ variant tend more to target mitochondria and had higher pro-apoptotic activity than the TP53 Pro72 variant during cellular stress response. ${ }^{8}$ Studies suggested that the common polymorphism was associated with body weight regulation and development of diabetic phenotypes. ${ }^{9}$ However, the relationship between TP53 Pro72Arg polymorphism and T2DM susceptibility remained controversial. ${ }^{10,11}$ Thus, whether the common polymorphism is an underlying risk factor for T2DM and related complications needs further studies in other populations. Given that mitochondria-targeted TP53 influences mtDNA damage repair, ${ }^{12}$ we postulated that the TP53 Arg72 variant could affect mitochondrial physiological function under hyperglycemic stress in T2DM patients, and might play a protective role in the development of diabetic complications.

The aim of the present study is to examine the association of the TP53 Pro72Arg polymorphism with T2DM risk and diabetic complication occurrence, and to explore the effect of this functional polymorphism on mtDNA genome maintenance. We conducted this case-control study in a Chinese Han population and investigated mtDNA content, mtDNA 4977 deletion and transcriptional level of the mitochondrial genes in the cells of T2DM patients. The results further demonstrated the $T P 53 \operatorname{Arg} 72$ variant was a genetic risk for T2DM, and the homozygous Arg/ $\mathrm{Arg}$ genotype of this locus was associated with mtDNA maintenance in the cells of T2DM patients which might lower risk for diabetic complications.

\section{Materials and Methods Study Subjects}

A total of 206 unrelated T2DM patients (mean age: $62.5 \pm$ 12.6 years old) were randomly recruited at the Second Affiliated Hospital of Nanjing Medical University from August 2017 to May 2018, and T2DM was diagnosed according to the criteria for classification and diagnosis of diabetes. ${ }^{13}$ The non-diabetic controls, matched for race, age and gender, were randomly selected from persons who came to this hospital for routine physical health checkup. Detailed interviews and laboratory data were made for each person, and subjects were recruited when their fasting blood glucose level was less than $6.1 \mathrm{mmol} / \mathrm{L}$, and who had no pharmacological treatment at time of the experimental session. The subjects, including the patients, were ruled out if they were suffering from acute inflammation, autoimmune diseases, tuberculosis, cancer, cardiovascular diseases. Eventually 446 Chinese non-diabetic individuals (mean age: $62.5 \pm 12.9$ years old) were recruited and served as the control group. Patient charts were reviewed, and the clinical characteristics, such as past disease history, family history of diabetes, body mass index (BMI), hemoglobin A1c (HbAlc) level and diabetes-related complications were recorded. For the complications, 
diabetic nephropathy was defined as a urinary albumin/ creatinine ratio of $30 \mathrm{mg} / \mathrm{g}$ or greater.

The study was approved by the local ethics committee of the Second Affiliated Hospital of Nanjing Medical University and was performed in accordance with the principles of the Declaration of Helsinki. All works on sample collection and processing were carried out with the informed consent from all subjects. Whole-blood sample was obtained from each subject in the morning after an overnight fast and collected in a citrate-anticoagulated tube.

\section{DNA Extraction and Genotyping}

Genomic DNA was extracted from the blood samples by a TIANamp Genomic DNA Kit (TIANGEN) and quantified by spectrophotometry. Genotyping of TP53 Pro72Arg was determined by a polymerase chain reaction-restriction fragment length polymorphism (PCR-RFLP) method with forward primer 5'-GTCAGATCCTAGCGTCGAGC-3' and reverse primer 5'- GCAAGAAGCCCAGACGGAAA-3', and followed by the Sanger sequencing of $10 \%$ samples with the heterozygous or homozygous variant (Figure S1). Sanger sequencing of PCR product was performed with the forward or reverse primer and BigDye Terminator v3.1 kit on ABI PRISM 3130 Genetic Analyzer (Applied Biosystems).

\section{Relative mtDNA Content Determination}

We chose two mtDNA fragments of MT-ND1 and MT-CO2 genes and a nuclear genomic fragment of $\beta$-2-microglobulin $(\beta 2 M)$ gene (primer sequences shown in Table $\mathrm{S} 1$ ), to reflect the levels of mtDNA content and nuclear genomic content, respectively. The mtDNA content was determined by fluorescence-based quantitative PCR (FQ-PCR) on ViiA Real-Time System (Applied Biosystems) with Power SYBR Green PCR Master Mix (Applied Biosystems). FQ-PCR was performed with $10 \mathrm{ng}$ of total DNA, and the PCR cycling condition was 1 cycle for $3 \mathrm{~min}$ at $95^{\circ} \mathrm{C}$, followed by 40 cycles for $5 \mathrm{~s}$ at $95^{\circ} \mathrm{C}$, and $30 \mathrm{~s}$ at $60^{\circ} \mathrm{C}$. The relative mtDNA content was estimated by calculating the ratio between mitochondrial fragment and nuclear fragment signals of each sample.

\section{MtDNA Deletion Analysis}

MtDNA with 4977 bp deletion mutation (mtDNA4977) is more common in cells with severe oxidative stress. The mtDNA4977 deletion, from nucleotide position (np) 8469 to $\mathrm{np} 13447$, was analyzed by PCR approach with 5'-
CCGGGGGTATACTACGGTCA-3' (forward) and 5'ATAGCTTTTCTAGTCAGGTT-3' (reverse) primers. PCR amplification was performed with Vazyme LAmp Master kit (Vazyme) as follows: $25 \mathrm{ng}$ total DNA in a reaction mix of $50 \mu \mathrm{L}$ with dNTPs at $200 \mu \mathrm{M}$ for each nucleotide, $1.5 \mathrm{mM}$ final concentration of $\mathrm{Mg}^{2+}, 20 \mathrm{pmol}$ each primer and 1.5 units of DNA polymerase, and PCR cycling condition was $5 \mathrm{~min}$ of initial denaturation at $94^{\circ} \mathrm{C}, 36$ cycles of denaturation $\left(94^{\circ} \mathrm{C}\right.$ for $\left.30 \mathrm{~s}\right)$, annealing $\left(58^{\circ} \mathrm{C}\right.$ for $\left.30 \mathrm{~s}\right)$ and extension $\left(72^{\circ} \mathrm{C}\right.$ for $\left.10 \mathrm{~min}\right)$, and a final $10 \mathrm{~min}$ of extension at $72^{\circ} \mathrm{C}$. Finally, the PCR products were separated on $0.8 \%$ agarose gels, and were either 894 bp fragment (4977 bp mtDNA deletion) or 536 bp fragment (5335 bp mtDNA deletion) or/and 5871 bp fragment (wild-type mtDNA) based on the type of deletion.

\section{MtDNA Transcription Analysis}

Reverse transcriptase-quantitative PCR (RT-qPCR) was used to assess the expression levels of mitochondrial genome in the cells of T2DM patients. Firstly, peripheral blood mononuclear cells (PBMCs) were isolated from patient's whole-blood samples using Ficoll Density Gradient. Total RNAs were extracted using TRIzol reagent (ambion by Life Technologies) and treated with 2 U DNase I. Then, $500 \mathrm{ng}$ of total RNAs were transcribed with All-in-One First-Strand cDNA Synthesis Kit according to the manufacturer's instruction, and the RT-qPCR assay was performed to quantify the gene expression of interest using the specific primers and Power SYBR Green PCR Master Mix (Applied Biosystems) on the ABI ViiA Real-Time System. The quantitative PCR cycling conditions consisted of one 3 -min cycle at $95^{\circ} \mathrm{C}$, followed by 40 cycles at $95^{\circ} \mathrm{C}$ for $5 \mathrm{~s}$ and $60^{\circ} \mathrm{C}$ for $30 \mathrm{~s}$. The primer sequences for the RT-qPCR experiment are listed in the Table S1.

Six mtDNA-encoded genes of interest were $M T$ RNR2, MT-CO1, MT-CO2, ATPase6, MT-ND1 and MT$C Y B$, and they were used to assess mtDNA transcriptional activity. Results of these mitochondrial gene expressions were quantified by the $2^{\Delta \mathrm{Ct}}$ method and normalized with $G A P D H$ expression. Each cDNA sample had triplicates, and all PCR reactions were independently triplicated to ensure the result reproducibility. The PCR amplification efficiency was validated, and the melting curve analysis was done to verify non-specific PCR products. 


\section{Statistical Analysis}

All statistical analyses were carried out by GraphPad Prism software (version 7.00). The categorical variables were analyzed using chi-square test, and the comparisons of numeric variables between two groups were conducted by a nonparametric Mann-Whitney test. Numeric variables in the three genotype groups were compared using ANOVA or Kruskal-Wallis test followed by a two-stage linear step-up method of Benjamini, Krieger and Yekutieli to correct for multiple comparisons with desired false discovery rate of 0.05 . Genotype distributions in diabetic and non-diabetic populations were tested by a goodness-of -fit chi-square test for departure from Hardy-Weinberg equilibrium (HWE). $P<0.05$ was considered statistically significant. Based on genotype frequency of the TP53 Pro72Arg polymorphism in the general Chinese Han population (data come from $\mathrm{CHB} / \mathrm{HCB}$ population of dbSNP build 151) and a small sampling of T2DM patients, the model of inheritance in the present study was considered as dominant model. Sample size of this study was estimated using free Power and Sample Size Calculator (http://powerandsamplesize.com/) with the variables set as follows: case-control study, sample ratio $=2$, and $80 \%$ power with type 1 error $\alpha=0.05$.

\section{Results}

\section{TP53 Arg72 Variants Have Different Association with Type 2 Diabetes and Its Complications}

A total of 206 T2DM and 446 non-diabetic participants enrolled in this study, and the baseline characteristics are shown in Table S2. The patients ranged from 36 to 90 years old with a median age of 63.0. Most of the patients $(53.4 \%, 110 / 206)$ were male, and the remaining $46.7 \%$ patients (96/206) were female (Table S2). Seen from the genotype distribution of TP53 Pro72Arg polymorphism in the patients, $11.7 \%(24 / 206)$ patients were homozygous for the Pro variant (Pro/Pro genotype), 30.0\% (62/206) patients were homozygous for the $\mathrm{Arg} 72$ variant ( $\mathrm{Arg} / \mathrm{Arg}$ genotype) and the rest $(58.3 \%, 120 / 206)$ were heterozygous (Pro/Arg genotype) (Table 1). In the control group, median age was 64.0 years old, and age ranged from 36 to 90 years old; 225 (50.4\%) controls were male (Table S2). Pro/Pro was detected in $84(18.8 \%)$, Pro/Arg in 240 (53.8\%) and $\mathrm{Arg} / \mathrm{Arg}$ in 122 (27.4\%) of the controls investigated (Table 1). Our data demonstrated a significant deviation to Hardy-Weinberg equilibrium in the T2DM
Table I The Distribution of TP53 Pro72Arg Polymorphism in the T2DM Patients and Non-Diabetic Controls

\begin{tabular}{|c|c|c|c|c|}
\hline $\begin{array}{l}\text { Genotype/ } \\
\text { Allele }\end{array}$ & $\begin{array}{c}\text { Patients, } \\
\begin{array}{c}N=206 \\
n(\%)\end{array}\end{array}$ & $\begin{array}{c}\text { Control, } \\
N=446 \\
n(\%)\end{array}$ & $P$ value & $\begin{array}{c}\text { OR } \\
(95 \% \mathrm{Cl})\end{array}$ \\
\hline Pro/Pro & 24 (II.7) & $84(18.8)$ & \multirow[t]{3}{*}{0.268} & \\
\hline Pro/Arg & $120(58.3)$ & $240(53.8)$ & & \\
\hline Arg/Arg & $62(30.0)$ & I $22(27.4)$ & & \\
\hline $\begin{array}{l}\text { Pro/Arg+Arg/ } \\
\text { Arg }\end{array}$ & I82 (88.3) & $362(8 \mid .2)$ & $\begin{array}{c}\mathbf{0 . 0 2 2} \\
\text { (vs Pro/ } \\
\text { Pro) }\end{array}$ & $\begin{array}{c}1.089 \\
(1.018- \\
1.164)\end{array}$ \\
\hline $\begin{array}{l}\text { Arg72 } \\
\text { variant }\end{array}$ & 0.592 & 0.543 & 0.093 & $\begin{array}{c}1.091 \\
(0.987- \\
1.207)\end{array}$ \\
\hline HWE test & $\begin{array}{l}\chi^{2}=8.75 \\
p=0.003\end{array}$ & $\begin{array}{l}\chi^{2}=3.15 \\
p=0.076\end{array}$ & & \\
\hline
\end{tabular}

Note: Bold type indicates a statistically significant difference $(p<0.05)$. Abbreviations: OR, odds ratio; $95 \% \mathrm{Cl}, 95 \%$ confidence interval; HWE, HardyWeinberg equilibrium.

patient population $\left(\chi^{2}=8.75, p=0.003\right)$ but not in the nondiabetic controls $\left(\chi^{2}=3.15, p=0.076\right)$ (Table 1$)$. The statistical analysis showed that there were no differences in genotype frequency between two groups $(p=0.268)$ (Table 1). However, TP53 Arg72 variant frequency in patients (59.2\%) was higher than that in controls $(54.3 \%)$, showing a borderline, but not significant, difference $(p=0.093)$ (Table 1). It was observed that the proportion of carriers with TP53 Arg72 variant (who had Pro/Arg or $A r g / A r g$ genotype) was higher in patients than that in controls $(88.3 \%$ versus $81.2 \%, p=0.022)$, and the OR was estimated to be 1.089 (95\% CI =1.018-1.164) (Table 1).

The subgroups of T2DM patients with different TP53 Pro72Arg genotypes were similar with respect to age (age at study and onset age) and sex distribution, diabetic duration, BMI, hypertension, positive family history, HbAlc levels, lipid profiles and insulin use $(p>0.05)$ (Table 2). In addition, prevalences of diabetic nephropathy were also similar among the three TP53 Pro72Arg genotype groups (Table 2 and Figure 1). However, we found that the prevalence of diabetic neuropathy in patients who harbored the $\mathrm{Arg} / \mathrm{Arg}$ genotype (6.5\%) was unexpectedly lower than in those who had the Pro/Pro genotype (25.0\%, $p=0.016)$ or heterozygous genotype $(18.3 \%, p=0.030)$ (Table 2 and Figure 1). We observed that patients with the Arg/Arg genotype had significantly decreased 
Table 2 Clinical Characteristics of T2DM Patients, Stratified According to the TP53 Pro72Arg Polymorphism

\begin{tabular}{|c|c|c|c|c|}
\hline & Pro/Pro & ProlArg & Arg/Arg & $P$ value \\
\hline Age at study (years old) & $59.8 \pm 13.1$ & $62.0 \pm 13.3$ & $64.5 \pm 10.8$ & 0.239 \\
\hline Onset age (years old) & $53.3 \pm 11.9$ & $52.2 \pm 12.3$ & $54.5 \pm 8.9$ & 0.455 \\
\hline Duration of diabetes (years) & $5(8)$ & 7 (II.5) & $8(29.5)$ & 0.198 \\
\hline Sex (Male/Female) & $10 / 14$ & $70 / 50$ & $30 / 32$ & 0.209 \\
\hline $\mathrm{BMI}\left(\mathrm{kg} / \mathrm{m}^{2}\right)$ & $24.9 \pm 3.24$ & $23.7 \pm 3.61$ & $24.5 \pm 3.63$ & 0.173 \\
\hline Hypertension, n (\%) & $13(54.2)$ & $53(44.2)$ & $29(46.8)$ & 0.667 \\
\hline Family history, $n$ (\%) & $7(3 \mid .8)$ & $30(26.1)$ & $19(32.2)$ & 0.656 \\
\hline $\mathrm{HbAc}(\%)$ & $9.39 \pm 1.68$ & $9.01 \pm 1.93$ & $9.02 \pm 1.66$ & 0.638 \\
\hline Triglyceride (mmol/L) & $2.51 \pm 2.17$ & $1.98 \pm 3.06$ & $2.54 \pm 2.78$ & 0.402 \\
\hline Total cholesterol (mmol/L) & $5.02 \pm 1.28$ & $4.97 \pm 1.03$ & $4.88 \pm 1.41$ & 0.451 \\
\hline HDL-cholesterol (mmol/L) & $1.36 \pm 0.53$ & $1.31 \pm 0.38$ & $1.33 \pm 0.45$ & 0.854 \\
\hline LDL-cholesterol (mmol/L) & $2.92 \pm 1.42$ & $3.06 \pm 1.21$ & $3.01 \pm 1.31$ & 0.877 \\
\hline Diabetic nephropathy, $n$ (\%) & $8(33.3)$ & $42(35.0)$ & $19(30.6)$ & 0.840 \\
\hline Diabetic neuropathy, n (\%) & $6(25.0)$ & $22(18.3)$ & $4(6.45)$ & 0.044 \\
\hline Diabetic retinopathy, $n$ (\%) & $7(29.2)$ & $36(31.0)$ & $9(14.8)$ & 0.058 \\
\hline Insulin use, $n(\%)$ & $17(70.8)$ & 87 (72.5) & $49(79.0)$ & 0.582 \\
\hline
\end{tabular}

Notes: Data are presented as the mean \pm standard deviation $(\mathrm{SD})$, median (quartile range) or number (percentage), and the bold type indicates a statistically significant difference $(p<0.05)$.

Abbreviations: BMI, body mass index; HbAc, hemoglobin AIc; HDL, high-density lipoprotein; LDL, low-density lipoprotein.

prevalence of diabetic retinopathy compared to those without (30.7\% versus $14.8 \%, p=0.018)$ (Table 2 and Figure 1$)$.

\section{TP53 Pro72Arg Polymorphism Is Associated with mtDNA Content and Not with the mtDNA4977 Deletion}

A comparison of mtDNA content showed significant differences among the three genotype groups $(p=0.002$ for MT-ND1 index and $p=0.004$ for MT-CO2 index) (Figure 2). The results illustrated that patients who carried Arg/Arg genotype had significantly increased level of mtDNA content than the heterozygotic ones (adjusted $p$ values were 0.002 and 0.008 for MT-ND1 and MT-CO2 indies, respectively). Compared to patients with Pro/Pro, those carrying $\mathrm{Arg} / \mathrm{Arg}$ genotype still had more mtDNA content, while it did not prove a statistical significance ( $p=0.200$ for $M T-N D 1$ index and $p=0.143$ for MT-CO2 index), and that might be because our small sample size was underpowered to show statistical differences between the two groups. Furthermore, we noted that the levels of mtDNA content for both the MT-ND1 and MT-CO2 indexes in patients with $\mathrm{Arg} / \mathrm{Arg}$ genotype were increased significantly, when compared with that in those without (the patients had Pro/Pro or Pro/Arg genotype) ( $p=0.002$ and $p=0.004$, respectively) (Figure 2).

In order to investigate whether the TP53 codon 72 polymorphism has an impact on the integrity of mitochondrial genome, we performed the analysis of common mtDNA4977 deletion in the blood cells of 206 T2DM patients using long-chain PCR approach. In this detection procedure, we failed to identify abnormal mtDNA sequence deletion regardless of the TP53 Pro 72 Arg genotypes (Figure 3). This showed that there is no correlation of this polymorphism with the common mtDNA4977 deletion in the progress of diabetes.

\section{Association of TP53 Pro72Arg Polymorphism with Transcriptional Activity of Mitochondrial Genome in the PBMCs of T2DM Patients}

We compared the expression levels of 6 mtDNA-encoded genes, MT-RNR2, MT-CO1, MT-CO2, ATPase6, MT-ND1 and MT-CYB, among the PBMCs of T2DM patients with different TP53 Pro 72 Arg genotypes, to explore the association of this polymorphism with transcriptional levels of mitochondrial genome. Statistically significant differences in the expression levels of MT-CO2, ATPase 6 and MTND1 genes have been discovered among the three genotype groups (Figure 4), and the results from multiple comparison tests revealed that the PBMCs from $\mathrm{Arg} / \mathrm{Arg}$ genotype patients had significantly higher mtRNA expression levels of MT-CO2, ATPase6 and MT-ND1 when compared to those from the Pro/Pro genotype patients, with adjusted $p$ values of $0.014,0.001$ and 0.007 , respectively, 


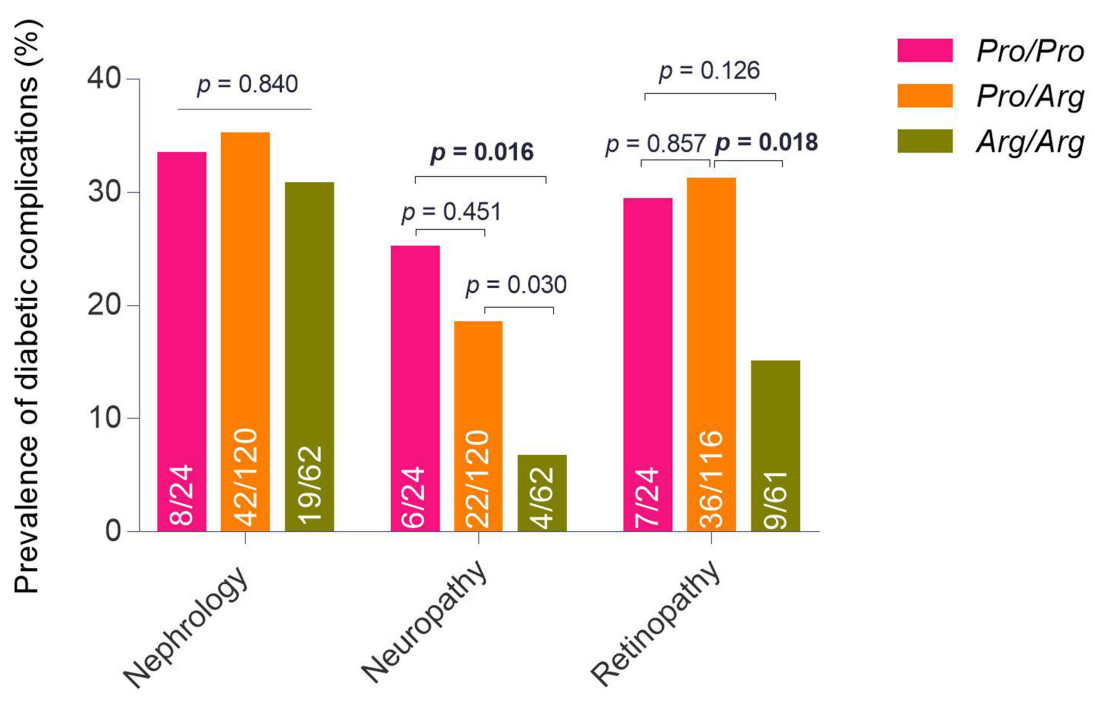

\begin{tabular}{|r|c|c|c|}
\hline Diabe tic complications & $\begin{array}{c}\text { Pro/Pro+Pro/Arg } \\
\text { (Pro carriers })\end{array}$ & Arg/Arg & P value \\
\hline Nephrology, n (\%) & $50(34.7)$ & $19(30.6)$ & 0.910 \\
\hline Neuropathy, n (\%) & $28(19.4)$ & $4(6.5)$ & $\mathbf{0 . 0 1 8}$ \\
\hline Retinopathy, n (\%) & $43(30.7)$ & $9(14.8)$ & $\mathbf{0 . 0 1 8}$ \\
\hline
\end{tabular}

Figure I The diabetic complication rates of T2DM patients stratified according to TP53 Pro72Arg polymorphism. Numbers within the bars represent number of observed patients with complications and total number of surveyed patients. Significant level in multiple comparisons among the three genotype groups was adjusted using Bonferroni correction, and the $p$ values in bold type were significant differences between the two groups.

A

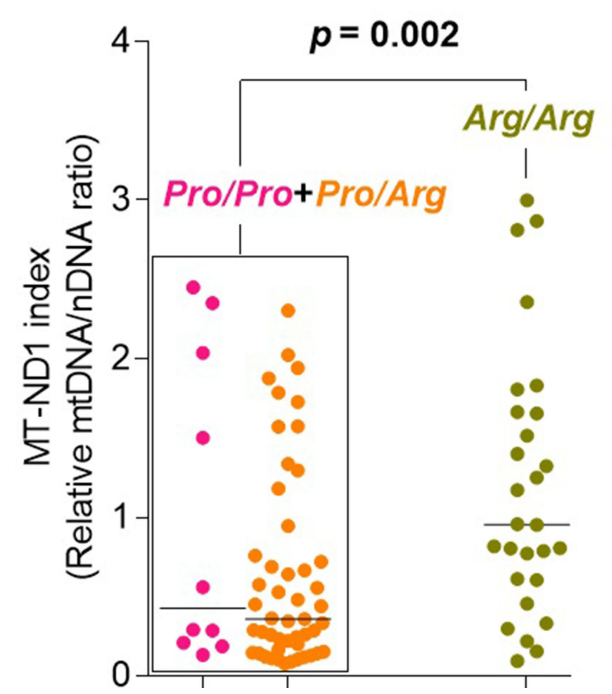

B $\quad p=0.004$

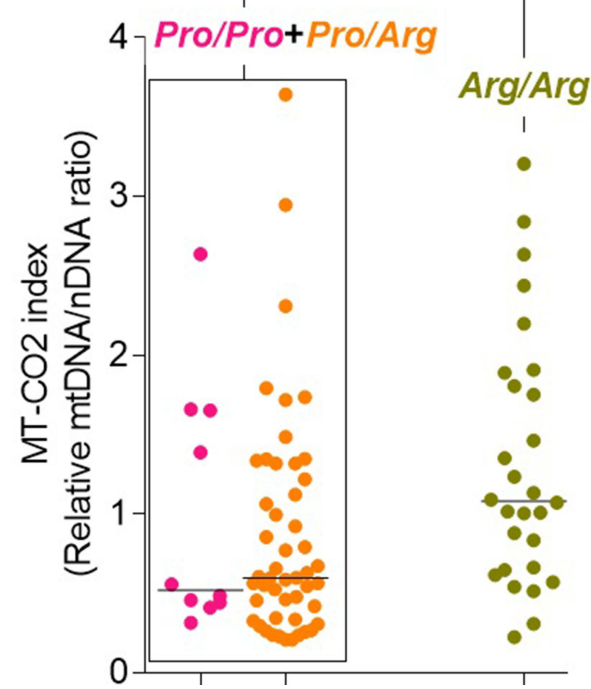

Figure 2 Association of the TP53 common polymorphism with mtDNA content in T2DM patients. Relative mtDNA content of the MT-NDI (A) and MT-CO2 (B) indexes in leukocytes isolated from T2DM patients. Each solid circle represents one checked sample of the mtDNA content analysis. Lines across the scatter dot represent the median. $p<0.01$ versus the merged group of Pro/Pro and Pro/Arg genotypes, by Mann-Whitney test.

or those from the Pro/Arg genotype patients, with adjusted $p$ values of $0.009,0.001$ and 0.007 , respectively (Figure 4). There were no statistically significant differences of $M T-R N R 2, M T-C O 1$ and $M T-C Y B$ gene expressions among the three genotype groups $(p=0.060$, 0.061 and 0.301 , respectively), but they did have the same 


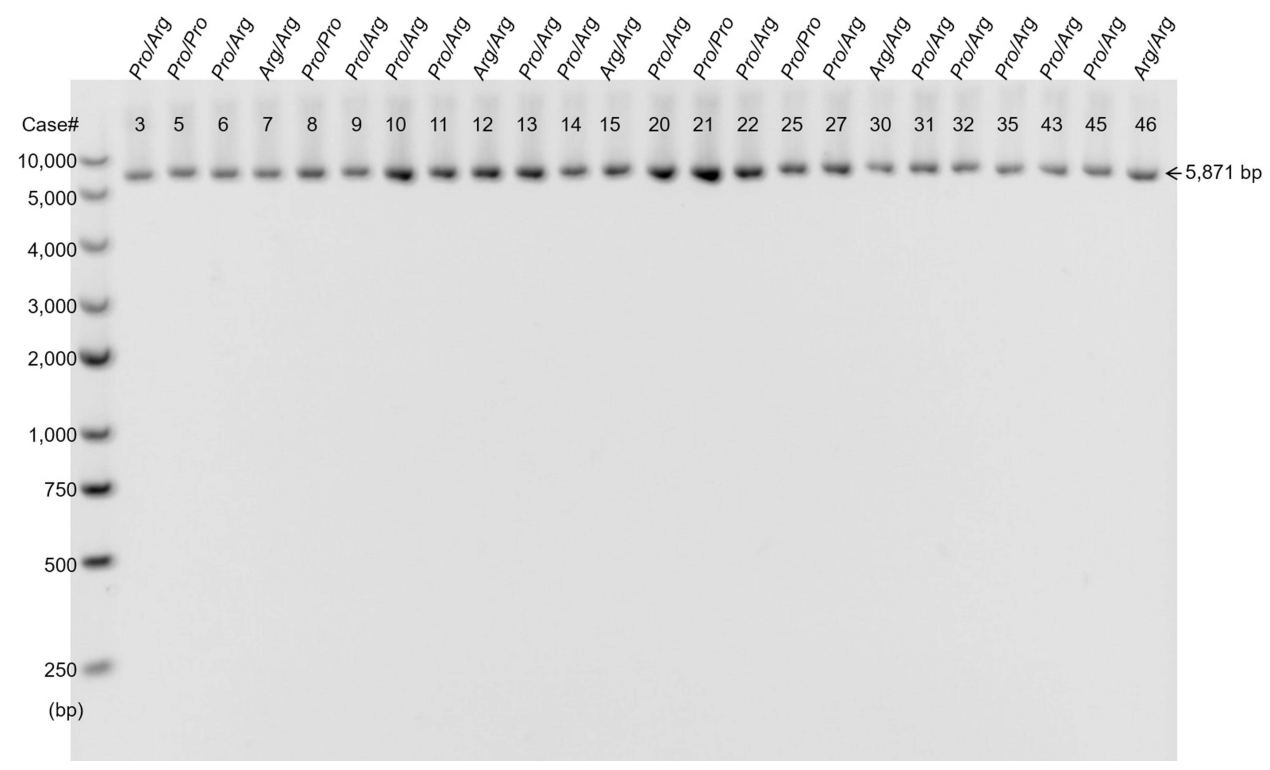

Figure 3 Representative result of ethidium bromide-stained agarose gel electrophoresis showed no abnormal mtDNA sequence deletion in mtDNA4977 deletion detection of leukocytes from T2DM patients. Genotypes of the TP53 Pro72Arg polymorphism and patient IDs are shown, respectively.

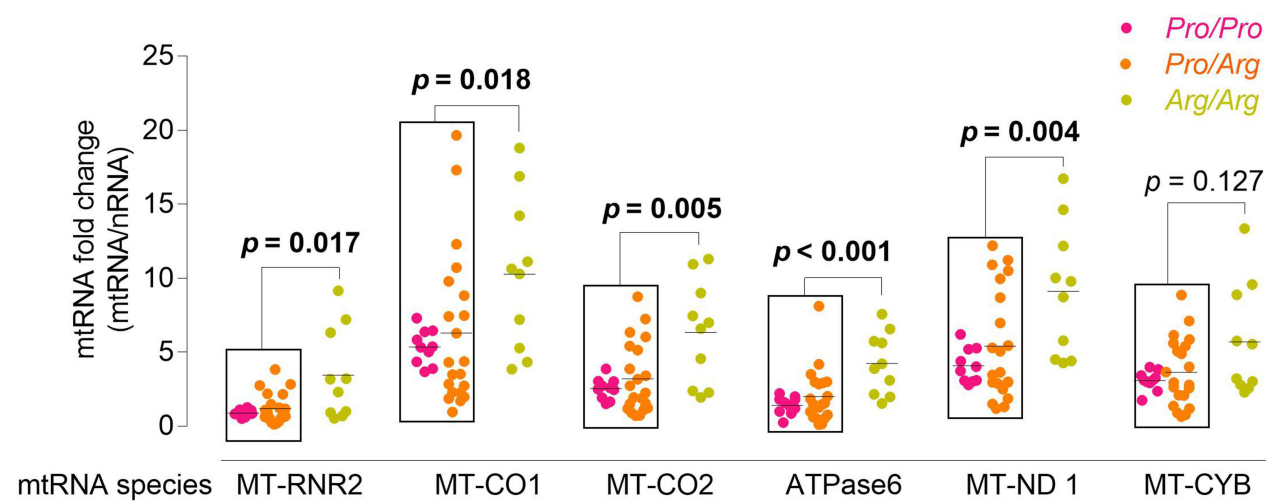

Figure 4 Association of the TP53 common polymorphism with mtRNA expressions in T2DM patients. Each solid circle represents one checked sample in RT-qPCR analysis. Lines across the scatter dot represent the median. The $p$ values in bold type were significant differences between the Arg/Arg group and the merged group of Pro/Pro and Pro/ Arg genotypes, by Mann-Whitney test.

trend, in which the highest expression levels of $M T-R N R 2$, MT-COI and MT-CYB genes were in the Arg/Arg group (Figure 4). Even more interestingly, in all surveyed genes but the $M T-C Y B$, the mtRNA expressions were relatively higher in the $\mathrm{Arg} / \mathrm{Arg}$ group compared with the merged group of Pro/Pro and Pro/Arg patients (Pro/Pro + Pro/Arg group) (Figure 4).

\section{Discussion}

The prevalence of type 2 diabetes has risen alarmingly worldwide. Multiple evidence points out TP53 has an important metabolic function and is required for homeostasis of lipid and glucose metabolism. ${ }^{14,15}$ These findings confirmed that aberrant TP53 function was correlated with development and advances of many metabolic diseases, ${ }^{15}$ especially obesity ${ }^{16}$ and diabetes. ${ }^{6}$ Study on a mouse model showed that inhibition of TP53 activity in adipose tissue markedly ameliorated senescence-like changes, reduced pro-inflammatory response and improved insulin resistance with diabetes-like disease. ${ }^{6}$ In ontology, TP53 is a key mediator of cellular response to DNA damage, and is activated by exposure to genotoxic or oxidative stress, resulting in DNA repair, cell-cycle arrest or apoptosis, and determining cell fate towards either survival or death. ${ }^{17}$ It primarily acts as a nuclear transcription factor and is activated by oxidative stress and DNA damage, participating in regulation of multiple cellular responses through the transcription-dependent 
pathways. ${ }^{17}$ Furthermore, TP53 protein can enter into mitochondria and participate in the cell death regulation and mtDNA maintenance. Studies have proved that TP53 protein can translocate to mitochondria in response to increased ROS and directly or indirectly facilitate mtDNA repair. ${ }^{12,18}$

A large-scale candidate gene analysis suggested that a common functional polymorphism, TP53 Pro72Arg, was significantly associated with susceptibility to type 2 diabetes. ${ }^{19}$ This association was replicated in other studies. ${ }^{20,21}$ However, some controversial research showed no significant differences of the incidence for metabolic and pre-diabetic phenotypes between homozygous TP53 Pro72 mice and homozygous TP53 Arg72 mice. $^{10}$ Therefore, we further explored the correlation between this polymorphism and risk of T2DM and its complications in a Han Chinese population-based case-control study. In the present study, we recruited 206 T2DM patients and 446 non-diabetic controls. The sample size was consistent with the theoretical sample size calculated based on the dominant inheritance model of the Arg72 risk variant in a Chinese Han population. We found that the different distribution of TP53 Pro72Arg genotypes (Pro/ Pro, Pro/Arg and Arg/Arg) was not statistically significant between the T2DM patients and the non-diabetic controls $(p=0.268)$ (Table 1). Similarly, the frequency of the allele was not statistically significant between the T2DM patients and the non-diabetic controls $(p=0.093)$ (Table 1). However, $88.3 \%$ of the patients carried the TP53 $\mathrm{Arg} 72$ variant (the $G$ allele of TP53 Pro72Arg locus, Arg/Arg and Pro/Arg genotypes), significantly higher than the $81.2 \%$ who carried them in the controls $(p=0.22$ ), and a significant deviation to Hardy-Weinberg equilibrium was found in patients $(p=0.003)$ (Table 1$)$. This different distribution of the TP53 Arg72 variant carriers between two groups supported that this variant might be a genetic risk factor for T2DM.

With regard to the relationship between the genotype of TP53 Pro72Arg polymorphism and clinical characteristics of T2DM patients, no significant difference was found in onset age, sex, obesity, hypertension, positive family history, $\mathrm{HbAlc}$ and lipid levels between the three genotype groups. It might be related to the variable interfering factor of clinical characteristics in diabetic patients such as hyperglycemia, eating habits or drug treatments. In order to further clarify the relation between genetic disposition and phenotypes, in addition to expanding the sample size of T2DM patients, the differences of those characteristics between genotype groups in the nondiabetic controls should be examined. However, we unexpectedly found that the TP53 $\operatorname{Arg} 72$ variant was significantly and negatively related to the development of diabetic complications (Figure 1). These results suggested that the homozygous TP53 $\operatorname{Arg} 72$ variant was associated with a decreased risk for diabetic-related complications and might be a protective factor in developing diabetic complications. One of the major etiological factors for diabetic complications is the continual hyperglycemia in the circulation of T2DM patients. ${ }^{1,22}$ The prolonged exposure to hyperglycemia is a potent inducer of reactive oxygen species (ROS) and causes excessive production of ROS through mitochondrial-related pathway in tissue cells. ${ }^{22}$ Excessive ROS can stimulate a series of biochemical changes, including cytokine dysregulation, abnormal apoptosis signal transduction, as well as considerable damage of biomacromolecules, that threaten physiological function of the host tissues and/or organs, especially in the nervous system and cardiovascular system. ${ }^{22}$ Of the ROSinduced damage to biomacromolecules, the DNA damage has been accumulating over time, which in severe cases can lead to a drastic DNA damage response (DDR) and finally result in cell cycle arrest, senescence and apoptosis of the tissue cells. ${ }^{23}$ DNA damage in mitochondrial genome is in a "feed-forward"-like loop, with ROS causing mtDNA damage, which in turn promotes ROS production by mitochondrial-related pathway. ${ }^{22}$ Thus, TP53 is involved in the maintenance of mitochondrial genome, which can in theory protect tissue cells against hyperglycemia-induced oxidative stress. This is approximately accordant to our results, and low prevalences of diabetic neuropathy and retinopathy were noted in patients with $\mathrm{Arg} / \mathrm{Arg}$ genotype. Furthermore, results from comparisons of mtDNA content and transcriptional levels among the three different genotypes showed that high levels of mtDNA content and mtDNA expression were associated with $\mathrm{Arg} / \mathrm{Arg}$ genotype. It supports a protective effect of TP53 $\operatorname{Arg} 72$ variant for mitochondrial function of tissue cells under hyperglycemia-induced oxidative stress. Thus, we postulated that the causes of low prevalence of diabetic complication in Arg/Arg genotype patients might be ascribed to a more stable mitochondrial genome with increased mtDNA transcriptional activity. The major drawback of this study is the small sample size. Thus, those results need further validation by correlating the clinical characteristics and the severity of diabetic- 
related complications with the TP53 Pro72Arg polymorphism in a large T2DM population.

\section{Conclusion}

Overall, our findings indicated that TP53 Pro72Arg polymorphism was a genetic risk factor of type 2 diabetes, and the TP53 $A r g 72$ variant was associated with lower risk of developing serious complications in the progress of type 2 diabetes. Further, the results from this study suggested that the TP53 Arg72 variant had a positive impact on mitochondrial maintenance, and was associated with higher mtDNA content and increased mtDNA transcriptional level, which would confer lower prevalence of complication in patients carrying this gene variant.

\section{Human and Animal Rights}

The experiment is mainly to explore an association of TP53 codon 72 polymorphism with type 2 diabetes mellitus and its complications in humans; the study was anonymous and only used currently existing medical data. The study was approved by the local ethics committee and was performed in accordance with the principles of the Declaration of Helsinki.

\section{Data Sharing Statement}

We would not share the biospecimen in this study, but the data used and/or analyzed during the experimental process are available from the corresponding authors (Wenwen Guo, hguowen@163.com and Yan Wang, wangyan_9999@sina.com) on reasonable request.

\section{Ethics Approval and Consent to Participate}

All works on sample collection and processing were carried out with the informed consent from all participants. These works were approved by the ethics committee of the Second Affiliated Hospital of Nanjing Medical University.

\section{Acknowledgment}

We are thankful to all the participants who enrolled in this study.

\section{Funding}

This work was supported by the National Natural Science Foundation of China (81501205), the Six Major Talent Summit of Jiangsu (2018-WSW-063), Youth Medical Talent of Jiangsu Province (QNRC2016665), and 789 Outstanding Talent Program of the Second Affiliated
Hospital of Nanjing Medical University (789ZYRC 202080122).

\section{Disclosure}

The authors declare that they have no conflicts of interest.

\section{References}

1. Schmidt AM. Highlighting diabetes mellitus: the epidemic continues. Arterioscler Thromb Vasc Biol. 2018;38(1):e1-e8. doi:10.1161/ ATVBAHA.117.310221

2. Taylor R. Type 2 diabetes: etiology and reversibility. Diabetes Care. 2013;36(4):1047-1055. doi:10.2337/dc12-1805

3. Javeed N, Matveyenko AV. Circadian etiology of type 2 diabetes mellitus. Physiology (Bethesda). 2018;33(2):138-150. doi:10.1152/ physiol.00003.2018

4. Alharbi KK, Abudawood M, Ali khan I. Amino-acid amendment of Arginine-325-Tryptophan in rs13266634 genetic polymorphism studies of the SLC30A8 gene with type 2 diabetes-mellitus patients featuring a positive family history in the Saudi population. J King Saud Univ Sci. 2021;33(1):101258. doi:10.1016/j.jksus.2020.101258

5. Ortega FJ, Moreno-Navarrete JM, Mayas D, et al. Inflammation and insulin resistance exert dual effects on adipose tissue tumor protein 53 expression. Int J Obes (Lond). 2014;38(5):737-745. doi:10.1038/ ijo.2013.163

6. Kung CP, Murphy ME. The role of the p53 tumor suppressor in metabolism and diabetes. $J$ Endocrinol. 2016;231(2):R61-R75. doi:10.1530/JOE-16-0324

7. Kodal JB, Vedel-Krogh S, Kobylecki CJ, Nordestgaard BG, Bojesen SE. TP53 Arg72Pro, mortality after cancer, and all-cause mortality in 105,200 individuals. Sci Rep. 2017;7(1):336. doi:10. 1038/s41598-017-00427-x

8. Dumont P, Leu JI, Della Pietra AC 3rd, George DL, Murphy M. The codon 72 polymorphic variants of $\mathrm{p} 53$ have markedly different apoptotic potential. Nat Genet. 2003;33(3):357-365. doi:10.1038/ng1093

9. Kung CP, Leu JI, Basu S, et al. The P72R polymorphism of p53 predisposes to obesity and metabolic dysfunction. Cell Rep. 2016;14 (10):2413-2425. doi:10.1016/j.celrep.2016.02.037

10. Reiling E, Speksnijder EN, Pronk AC, et al. Human TP53 polymorphism (rs1042522) modelled in mouse does not affect glucose metabolism and body composition. Sci Rep. 2014;4:4091. doi:10.1038/srep04091

11. Molchadsky A, Ezra O, Amendola PG, et al. p53 is required for brown adipogenic differentiation and has a protective role against diet-induced obesity. Cell Death Differ. 2013;20(5):774-783. doi:10.1038/cdd.2013.9

12. Park JH, Zhuang J, Li J, Hwang PM. p53 as guardian of the mitochondrial genome. FEBS Lett. 2016;590(7):924-934. doi:10.1002/ 1873-3468.12061

13. American Diabetes Association. 2. Classification and diagnosis of diabetes: standards of medical care in diabetes-2019. Diabetes Care. 2019;42(Suppl 1):S13-S28. doi:10.2337/dc19-S002

14. Lacroix M, Riscal R, Arena G, Linares LK, Le Cam L. Metabolic functions of the tumor suppressor p53: implications in normal physiology, metabolic disorders, and cancer. Mol Metab. 2020;33:2-22. doi:10.1016/j.molmet.2019.10.002

15. Kang JG, Lago CU, Lee JE, et al. A mouse Homolog of a Human TP53 Germline mutation reveals a lipolytic activity of p53. Cell Rep. 2020;30(3):783-792.e785. doi:10.1016/j.celrep.2019.12.074

16. Krstic J, Reinisch I, Schupp M, Schulz TJ, Prokesch A. p53 functions in adipose tissue metabolism and homeostasis. Int J Mol Sci. 2018;19 (9):2622. doi:10.3390/ijms19092622 
17. Joruiz SM, Beck JA, Horikawa I, Harris CC. The $\Delta 133 p 53$ Isoforms, Tuners of the p53 Pathway. Cancers (Basel). 2020;12(11):3422. doi: $10.3390 /$ cancers 12113422

18. Kazak L, Reyes A, Holt IJ. Minimizing the damage: repair pathways keep mitochondrial DNA intact. Nat Rev Mol Cell Biol. 2012;13 (10):659-671. doi:10.1038/nrm3439

19. Gaulton KJ, Willer CJ, Li Y, et al. Comprehensive association study of type 2 diabetes and related quantitative traits with 222 candidate genes. Diabetes. 2008;57(11):3136-3144. doi:10.2337/db07-1731

20. Burgdorf KS, Grarup N, Justesen JM, et al. Studies of the association of Arg72Pro of tumor suppressor protein p53 with type 2 diabetes in a combined analysis of 55,521 Europeans. PLoS One. 2011;6(1): e15813. doi:10.1371/journal.pone. 0015813
21. Sliwinska A, Kasznicki J, Kosmalski M, et al. Tumour protein 53 is linked with type 2 diabetes mellitus. Indian J Med Res. 2017;146 (2):237-243. doi:10.4103/ijmr.IJMR_1401_15

22. Kaludercic N, Di Lisa F. Mitochondrial ROS formation in the pathogenesis of diabetic cardiomyopathy. Front Cardiovasc Med. 2020;7:12. doi:10.3389/fcvm.2020.00012

23. Tatsch E, De Carvalho JA, Hausen BS, et al. Oxidative DNA damage is associated with inflammatory response, insulin resistance and microvascular complications in type 2 diabetes. Mutat Res. 2015;782:17-22. doi:10.1016/j.mrfmmm.2015.10.003

\section{Publish your work in this journal}

The International Journal of General Medicine is an international, peer-reviewed open-access journal that focuses on general and internal medicine, pathogenesis, epidemiology, diagnosis, monitoring and treatment protocols. The journal is characterized by the rapid reporting of reviews, original research and clinical studies across all disease areas. The manuscript management system is completely online and includes a very quick and fair peer-review system, which is all easy to use. Visit http://www.dovepress.com/ testimonials.php to read real quotes from published authors. 